\title{
Early Rehospitalization Post-Kidney Transplant Due to Infectious Complications: Can We Predict the Patients at Risk?
}

\author{
R. Leal ${ }^{a}$, H. Pinto ${ }^{a, \star}$, A. Galvão ${ }^{a}$, L. Rodrigues ${ }^{a}$, L. Santos $^{a}$, C. Romãozinho ${ }^{a}$, F. Macário $^{a}$, R. Alves ${ }^{a}$, \\ M. Campos ${ }^{a}, A$. Mota $^{b}$, and A. Figueiredo ${ }^{b}$
}

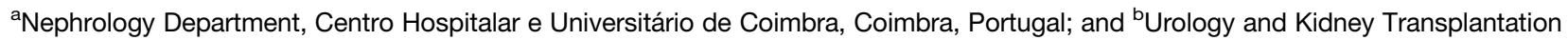
Department, Centro Hospitalar e Universitário de Coimbra, Coimbra, Portugal

\begin{abstract}
Introduction. Rehospitalization early post-kidney transplant is common and has a negative impact in morbidity, graft survival, and health costs. Infection is one the most common causes, and identifying the risk factors for early readmission due to infectious complications may guide a preventive program and improve outcome. The aim of this study was to evaluate the incidence, characterize the population, and identify the risk factors associated with early readmission for infectious complications post-kidney transplantation.

Methods. We performed a retrospective cohort study of all the kidney transplants performed during 2015. The primary outcome was readmission in the first 3 months posttransplant due to infectious causes defined by clinical and laboratory parameters.

Results. We evaluated 141 kidney transplants; $71 \%$ of subjects were men, with an overall mean age of $50.8 \pm 15.4$ years. Prior to transplant, $98 \%$ of the patients were dialysis dependent and $2 \%$ underwent pre-emptive living donor kidney transplant. The global readmission rate was $49 \%$, of which $65 \%$ were for infectious complications. The most frequent infection was urinary tract infection $(\mathrm{n}=28,62 \%)$ and the most common agent detected by blood and urine cultures was Klebsiella pneumonia $(\mathrm{n}=18,40 \%)$. The risk factors significantly associated with readmission were higher body mass index $(P=.03)$, diabetes mellitus $(P=.02)$, older donor $(P=.007)$, and longer cold ischemia time $(P=.04)$. There were 3 graft losses, but none due to infectious complications.

Conclusion. There was a high incidence of early rehospitalization due to infectious complications, especially urinary tract infections to nosocomial agents. The risk factors identified were similar to other series.
\end{abstract}

$\mathbf{H}$ OSPITAL readmission in early post kidney transplant (KT) period is frequent and nearly one-third of KT recipients are rehospitalized within 30 days of discharge $[1,2]$. Early rehospitalization (ERH) is associated with increased morbidity, transition-of-care errors, and costs both to patients and the health care system $[3,4]$. One of the most common causes for ERH is infection, and KT recipients have a cumulative incidence of infections of over $75 \%$ in the first year $[3,5,6]$. The high incidence of infection is multifactorial and related in part to the surgical procedure, immunosuppressive drugs, exposure to nosocomial pathogens, and necessity for devices such as urinary catheters and intravascular lines [7].
Despite improvements in immunosuppressive therapy and surgical techniques, infectious complications remain a major cause of morbidity, and these complications, especially urinary tract infection (UTI), have been associated with increased risk for graft rejection and general worst graft outcome $[1,7,8]$.

The aims of this study were to evaluate the incidence of infectious complications in the first 3 months post-KT, characterize the population- and transplant-elated variables, and identify risk factors associated with ERH.

*Address correspondence to Praceta Prof. Mota Pinto, 3000075 Coimbra, Portugal. E-mail: helenasofiapinto@gmail.com 


\section{Patients and Methods}

We performed a retrospective, single-center cohort study of all the 141 KTs performed during 2015 in our center. Follow-up was until March 2016, which included a posttransplant time range of 3 to 15 months.

All patients were followed up according to our center surveillance protocol: medical and nurse visit twice a week for the first month and once a week at the second and third months post-transplant. At every visit, blood tests for graft function, urinalysis, and hemogram are obtained. At the end of the third month, cytomegalovirus (CMV), Epstein-Barr virus, and donor-specific antibodies are searched in every patient. If dysfunction occurs during the time of follow-up, patients receive blood tests for $\mathrm{CMV}$, Epstein bar virus, and donorspecific antibodies and Doppler ultrasound. If an infection is suspected, blood and urinary cultures are performed.

The primary outcome was hospital readmission due to infectious causes in the first 3 months post-transplant. The diagnosis of infection was defined based on clinical features at presentation, laboratory parameters (positive biological fluid cultures, CMV detection by viral proteins or isolation of the virus in the blood, C-reaction protein levels, procalcitonin, and leukocytes), and radiology exams. Demographic and baseline characteristics of the study population including donor, recipient, and transplantrelated variables were abstracted from patient records.

Data were analyzed using SPSS statistics software version 15 Windows (SPSS, Inc, Chicago, Ill, USA). Comparisons used the $\chi^{2}$ test for categorical variables and Student $t$ test or the Mann-Whitney $U$ test for continuous variables, as appropriate. To measure odds ratios of categorical data and their significance, we used the cross-tabs method with risk estimation; for continuous variables, we used binary regression. All tests were 2 tailed; $P<.05$ was considered significant.

\section{RESULTS}

Demographic and Transplant Characteristics of the Study Population

We evaluated $141 \mathrm{KTs}$ during the study period, including $71 \%(\mathrm{n}=100)$ male recipients, $96 \%(\mathrm{n}=135)$ Caucasians, with an overall mean age of $50.8 \pm 15.4$ years. Prior to transplant, $98 \%$ of the patients performed dialysis $(79 \%$ hemodialysis and $19 \%$ peritoneal dialysis) with a mean length of time of dialysis of $44.6 \pm 28.9$ months, and 3 patients $(2 \%)$ underwent pre-emptive living donor KT. It was the first transplant in $92 \%$ of the patients $(\mathrm{n}=130)$, and $95 \%(\mathrm{n}=134)$ received a cadaveric graft. Regarding immunosuppression, induction with basiliximab was performed in $84 \%$ of the recipients $(\mathrm{n}=118)$, and maintenance therapy with calcineurin inhibitors was used in $94 \%$ patients $(\mathrm{n}=133)$.

\section{Readmission Rate and Infection}

Overall, 69 patients were readmitted in the first 3 months post-transplant, representing a readmission rate of $49 \%$.
Among them, 28 (41\%) patients had 1 and 41 (59\%) had 2 or more readmissions. Infection was the main cause for ERH, affecting 45 patients $(45 / 69,65 \%)$, followed by graft dysfunction $(13 / 69,19 \%)$ and surgical complications $(11 / 69,16 \%)$.

The main site of infection was the urinary tract $(n=28)$, followed by surgery-related infections $(n=8)$, infection of the dialysis access $(n=3)$, respiratory tract infection $(\mathrm{n}=2)$, gastrointestinal infection $(\mathrm{n}=2)$, and CMV infection $(\mathrm{n}=2)$. Klebsiella pneumoniae was the most frequently isolated micro-organism $(\mathrm{n}=18)$, followed by Pseudomonas aeruginosa $(\mathrm{n}=5)$, Escherichia coli $(\mathrm{n}=3)$, and Enterococcus faecium $(\mathrm{n}=2)$. In one patient with respiratory tract infection, Aspergillus fumigatus was isolated. In 14 patients, no agent was isolated. The mean duration of antimicrobial therapy was $12.5 \pm 7$ days.

\section{Predictors of Early Rehospitalization Due to Infectious Complications}

The risk factors that were significantly associated with readmission for infectious causes in the first 3 months posttransplant were higher body mass index (26.3 vs $24.4, P=.03$; odds ratio $[\mathrm{OR}]=1.254,95 \%$ confidence interval [1.09-1.348], $P<.05)$, the presence of diabetes mellitus (DM) $(\mathrm{OR}=3.27$ [1.1-9.6], $P<.05$ ), older donor (56.5 vs 51.2, $P=.007$, $\mathrm{OR}=1.03$ per year [1.02-1.063], $P<.05)$, and longer cold ischemia time $(19.0$ vs $16.9 ; P=.04, \mathrm{OR}=1.7$ per hour [1.04-2.67], $P<.05$; Table 1$)$.

\section{Follow-up}

Time of follow-up varied between 3 and 15 months, and no graft lost or death was registered. The mean value of serum creatinine at follow-up was $1.65 \pm 0.42 \mathrm{mg} / \mathrm{dL}$.

Table 1. Epidemiology and Risk Factors for Rehospitalization Due to Infectious Complications During the First 3 Months Post-Kidney Transplant

\begin{tabular}{|c|c|c|c|}
\hline & Rehospitalized & $\begin{array}{c}\text { Not } \\
\text { Rehospitalized }\end{array}$ & $P$ \\
\hline \multicolumn{4}{|l|}{ Recipient characteristics } \\
\hline Age $(y)$ & $52.2 \pm 18$ & $49.4 \pm 13.4$ & NS \\
\hline Gender, female (n) & 19 & 22 & NS \\
\hline Body mass index $\left(\mathrm{kg} / \mathrm{m}^{2}\right)$ & $26.3 \pm 3.8$ & $24.4 \pm 3.7$ & .02 \\
\hline Dialysis duration (mo) & $44.9 \pm 23$ & $43.8 \pm 29$ & NS \\
\hline Diabetes mellitus, yes (n) & 11 & 6 & .03 \\
\hline Urological pathology, yes (n) & 12 & 13 & NS \\
\hline \multicolumn{4}{|l|}{ Transplant characteristics } \\
\hline Donor age (y) & $56.5 \pm 8.7$ & $51.2 \pm 11.3$ & .007 \\
\hline Living donor, yes (n) & 2 & 5 & NS \\
\hline Retransplant, yes (n) & 5 & 6 & NS \\
\hline HLA mismatches (mean) & $3.0 \pm 1.2$ & $3.2 \pm 1.1$ & NS \\
\hline Cold ischemia time (h) & $18.8 \pm 4.7$ & $16.8 \pm 6.2$ & .04 \\
\hline $\begin{array}{l}\text { Surgical complications } \\
\quad \text { during transplantation }(\mathrm{n})\end{array}$ & 8 & 9 & NS \\
\hline Urinary catheterization (d) & $6.5 \pm 3.2$ & $6.1 \pm 2.1$ & NS \\
\hline Delayed graft function $(n)$ & 7 & 8 & NS \\
\hline
\end{tabular}

Abbreviation: NS, not significant. 


\section{DISCUSSION}

Readmission in early post-KT period is frequent, and studies report frequencies of up to $40 \%$ in the first year, with higher rates in the first 6 months $[1,2,9]$. Our primary outcome was defined at 3 months post-KT because, in our center, this is the period considered of higher risk for early complications and patients have more frequent hospital visits.

In our study, the global rate of EHR was $49 \%$, and the main causes for readmission were infection, graft dysfunction, and surgical complications, which is similar to data present in the literature [3]. Of all the above, infectious complications were the most frequent reason for HER, representing $65 \%(n=45)$ of all the readmissions and $32 \%$ in the general study population. This tendency of increased infectious events over surgical complications and graft dysfunction has being noticed in the past years [5,6]. Advances in immunosuppression have led to more effective drugs that reduce the rate of acute rejection and consequently graft dysfunction but with the downside of increased infection risk $[10,11]$. The lower incidence of surgical complications may be partly due to improvements in surgical transplant techniques [7]. Despite that, in our sample, 8 patients had concomitant infection and surgical complications, a common feature in KT [12].

The main cause of infection in our study was UTI (62\%), consistent with literature data [13]. The higher susceptibility of UTI in KT recipients is multifactorial: they underwent a urological surgical procedure, present an immunosuppressed status, and have higher contact with nosocomial pathogens and invasive devices such as intravascular lines and urinary catheters [7].

There is controversy regarding urinary catheter removal: on the one hand, it is needed for maintaining urine drainage and to prevent of early obstruction, urinary leaks, and vesical fistulas and to better monitor diuresis [14]; on the other hand, it has been demonstrated that the duration of urethral catheterization is one of the most important risk factor for UTI, and advantages of early removal of the catheter have been emphasized [15]. The same controversy is present regarding double $\mathrm{J}$ ureteric stents: they decrease fistulas, hematuria, and stenosis rates but increase infection risk [16]. In our center, the mean time for urinary catheterization is $6.3 \pm 2.7$ days, and there was no significant difference between groups (Table 1).

The main risk factors for EHR due to infection identified in our study were higher body mass index, the presence of DM, older donor, and longer cold ischemia time. Obese recipients have higher rates of prolonged length of hospital stay, wound dehiscence, and de novo post-transplant DM, and all of these factors are related to increased risk of infection [17]. DM is associated with earlier readmission in several studies due to various causes, including infection [5,18]. Patients with DM are more likely to have several other comorbidities, making them a highly vulnerable patient population. Patients who receive grafts from extended criteria donors have significant greater risk of
post-KT infection [19], which can be related to both donor and receptor, regarding the paradigm of old-for-old transplantation. Longer cold ischemia time triggers a cascade of noxious effects that increase inflammatory and immune responses that potentially result in worst graft and patient outcomes [20]. The exacerbated immune-inflammatory response in patients with longer cold ischemia time may induce the rescue of inflammatory cells, increasing the risk of infection in KT recipients [10].

Unplanned readmissions in early post-KT is associated with worse short- and long-term clinical outcomes, especially frequent rehospitalizations [4]. Although our study presented a short follow-up, no graft or patient loss was registered.

\section{CONCLUSION}

In our study, readmission in the first 3 months posttransplant due to infectious complications occurred in $32 \%$ of the recipients, and the risk factors were higher body mass index, the presence of DM, older donor, and longer cold ischemia time. Recognizing modifiable risk factors is of major importance regarding graft and patient outcome. KT transplant guidelines already take into account the prevention of the most common infections in the early post-KT period, including the prophylaxis of CMV infection and Pneumocystis carinii [21]. Patients identified as vulnerable from ERH due to infection may benefit from additional or different forms of monitoring and antibiotic therapy management.

\section{REFERENCES}

[1] McAdams-Demarco M, Grams M, Hall E, Coresh J, Segev D. Early hospital readmission after kidney transplantation: patient and center-level associations. Am J Transplant 2012;12:3283-8.

[2] McAdams-DeMarco M, Law A, Salter M, Chow E, Grams M, Walston J, et al. Frailty and early hospital readmission after kidney transplantation. Am J Transplant 2013;13:2091-5.

[3] Harhay M, Lin E, Pai A, Harhay M, Huverserian A, Mussell A, et al. Early rehospitalization after kidney transplantation: assessing preventability and prognosis. Am J Transplant 2013;13:3164-72.

[4] Luan F, Barrantes F, Roth R, Samaniego M. Early hospital readmissions post-kidney transplantation are associated with inferior clinical outcomes. Clin Transplant 2014;28:487-93.

[5] Boubaker K, Harzallah M, Ounissi M, Becha M, Guergueh T, Hedri H, et al. Rehospitalization after kidney transplantation during the first year: length, causes and relationship with long-term patient and graft survival. Transplant Proc 2011;43:1742-6.

[6] Moghani Lankarani M, Noorbala M, Assari S. Causes of re-hospitalization in different post kidney transplant periods. Ann Transplant 2009;14:14-8.

[7] Maraha B, Bonten H, van Hooff H, Fiolet H, Buiting A, Stobberingh E. Infectious complications and antibiotic use in renal transplant recipients during a 1-year follow-up. Clin Microbiol Infect 2001;7:619-25.

[8] Li A, Lam N, Naylor K, Garg A, Knoll G, Kim S. Early hospital readmissions after transplantation: burden, causes, and consequences. Transplantation 2016;100:713-8.

[9] Englesbe M, Dimick J, Fan Z, Baser O, Birkmeyer J. Case mix, quality and high-cost kidney transplant patients. Am J Transplant 2009;9:1108-14. 
[10] Sá H, Leal R, Rosa M. Renal transplant immunology in the last 20 years: a revolution towards graft and patient survival improvement. Int Rev Immunol 2016;28:1-29.

[11] Karuthu S, Blumberg E. Common infections in kidney transplant recipients. Clin J Am Soc Nephrol 2012;7:2058-70.

[12] Humar A, Matas A. Surgical complications after kidney transplantation. Semin Dial 2005;18:505.

[13] Sorto R, Irizar S, Delgadillo G, Alberu J, Correa-Rotter R, Morales-Buenrostro L. Risk factors for urinary tract infections during the first year after kidney transplantation. Transplant Proc 2009;42:280-1.

[14] Sagalowsky A, Ransler C, Peters P, et al. Urologic complications in 505 renal transplants with early catheter removal. J Urol 1983;129:86-124.

[15] Guler S, Cimen S, Hurton S, Molinari M. Risks and benefits of early catheter removal after renal transplantation. Transplant Proc 2015;47:2855-9.
[16] Akoh J, Rana T. Effect of ureteric stents on urological infection and graft function following renal transplantation. World J Transplant 2013;24:1-6.

[17] Gusukuma L, Harada K, Baptista A, Alencar M, de Sandes-Freitas T, Tedesco-Silva HJ, et al. Outcomes in obese kidney transplant recipients. Transplant Proc 2014;46:3416-9.

[18] Ramezani M, Ghoddousi K, Hashemi M, et al. Diabetes as the cause of end-stage renal disease affects the pattern of post kidney transplant rehospitalizations. Transplant Proc 2007;39:966.

[19] Barba J, Zudaire J, Robles J, et al. Complications of kidney transplantation with grafts from expanded criteria donors. World J Urol 2013;31:893.

[20] Ponticelli C. The impact of cold ischemia time on renal transplant outcome. Kidney Int 2015;87:272-5.

[21] Group KDIGO(TW). KDIGO clinical practice guideline for the care of kidney transplant recipients. Am J Transplant 2009;9: S1-155. 